\title{
"Son asimilados": Mayra Montero vis-à-vis Tomás Blanco y el discurso racial en Puerto Rico
}

\author{
Violeta Lorenzo Feliciano • University of Arkansas
}

\begin{abstract}
Resumen
El propósito en este trabajo es analizar "El entierro de Chianita: un complot chino" de Mayra Montero vis-à-vis el ensayo El prejuicio racial en Puerto Rico de Tomás Blanco con el fin de subrayar cómo el discurso racial de la Generación del 30 en Puerto Rico pervive en la actualidad y, entre otros asuntos, escamotea proyectos antirracistas. La primera parte de este trabajo analizará los ejemplos de la ópera y del teatro bufo que Montero incluye en su ensayo para justificar la presencia en el Puerto Rico del siglo XXI de personajes negros interpretados por artistas blancos que se oscurecen el rostro de negro (i.e. "blackface”). Dicho análisis usará premisas de teorías postcoloniales como punto de partida. La segunda parte estudiará las conexiones entre el ensayo de Blanco y el de Montero y mencionará posibles estrategias que ayuden a combatir el racismo institucionalizado.
\end{abstract}

$\mathrm{E}$ $\mathrm{n}$ el 2016, poco antes de las elecciones gubernamentales en Puerto Rico, la actriz Ángela Meyer intentó traer a la televisión puertorriqueña a Chianita, un personaje que ella popularizó en los años setenta. Este personaje tuvo etapas en las cuales sus reacciones exageradas, su mal gusto al vestir, su falta de educación, su mala pronunciación y uso pobre del lenguaje lo caracterizaban (Rivero 93). Eventualmente, el personaje fue reconstruido para representar a una jíbara inteligente que estaba al tanto de los problemas políticos y sociales a nivel local e internacional (Rivero 101). Su canción “Chianita gobernadora” gozó de gran popularidad puesto que criticaba el machismo y la inefectividad gubernamental. Asimismo, en sus apariciones televisivas Chianita discutía los temas de importancia actual, toda vez que se convirtió en portavoz de algunos ciudadanos: "if a neighborhood did not have electricity for months, and the governor did not respond to the citizens' complaints, they went to Chianita's television segment and both the character and the individuals demanded action" (Rivero 101). Así pues, no es extraño que en época de elecciones y ante un sinnúmero de problemas sociales, políticos y económicos que aquejan a los puertorriqueños Meyer intentara revivir al personaje en cuestión. ${ }^{1}$

Poco después de Meyer hacer pública su intención de revivir a Chianita, un grupo de activistas celebró un evento donde se enterró simbólicamente al personaje. La polémica principal en torno a Chianita estriba en que ésta es un personaje negro y Meyer es una actriz blanca que debe oscurecerse el rostro para interpretarlo. Los activistas del entierro forman parte de grupos antirracistas que denuncian-entre otros asuntos-la práctica por parte de artistas blancos de pintarse el rostro con el fin de interpretar personajes negros. Dicha práctica ha sido denigrante para los afrodescendientes debido a que los caricaturiza y presenta su color de piel como una anomalía que sirve para entretener. Además, el hecho de que en algunas representaciones Chianita no se exprese correctamente perpetúa estereotipos que asumen que las personas negras son tontas e incultas. Por último, darle lugar a un artista blanco para que represente a un personaje negro les cierra las puertas a artistas negros que suelen ser discriminados en el ámbito de las artes dramáticas (Rivero 10-12). Por estas razones los activistas que enterraron a Chianita entienden que es innecesario revivir a un personaje que dejó de salir en la televisión boricua a mediados de los ochenta y que realmente no tiene nada nuevo ni constructivo que aportar.

Las protestas en contra de Chianita no son nuevas. En su estudio Tuning Out Blackness Yeidy M. Rivero explica que entre 1973 y 1974 artistas como Sylvia del Villard y Carmen Belén Richardson criticaron a Chianita por su "blackface" o cara pintada de negro. En ese entonces Meyer defendió a su personaje aludiendo a la popularidad de éste entre el público 
afrodescendiente y a la escasez de artistas negros que pudieran salir en programas televisivos (Rivero 96). ${ }^{2}$ La controversia suscitó dimes y diretes ya que algunos apoyaban a Meyer y otros a del Villard (Rivero 99). Sin embargo, en aquella ocasión las críticas no tuvieron un gran impacto y Chianita mantuvo su presencia mediática.

Tal y como sucedió en la disputa entre Meyer y del Villard de 1973-1974, el entierro de 2016 recibió encomio de las personas que apoyan la lucha contra el racismo y reproches de las que no se percatan del racismo que hay detrás del "blackface". No obstante, la protesta de 2016 y los comentarios que recibió Meyer a través de las redes sociales surtieron efecto: Univisión decidió no incorporar al personaje en su programación y Meyer reticentemente aceptó que no valía la pena ofender a nadie. ${ }^{3}$ Ahora bien, justo cuando parecía que la controversia había acabado, la escritora Mayra Montero reabrió el debate en un corto texto que publicó en la columna ensayística que tiene en el periódico El Nuevo Día. En el mismo, Montero regaña a los activistas y justifica-a través de ejemplos de otras representaciones artísticas-la existencia de personajes negros interpretados por actores blancos con el rostro oscurecido.

Propongo que analizar el ensayo de Montero es neesario al hablar de lo racial en Puerto Rico, especialmente porque de su texto se desprende que denunciar el racismo en ocasiones implica tener una mentalidad colonizada que importa paradigmas raciales de Estados Unidos. Este enfoque es problemático porque establece una comparación entre Estados Unidos y Puerto Rico que socava la crítica constructiva y la búsqueda de soluciones al discrimen racial, pero además, es problemático porque repite parte del discurso racial de la llamada Generación del 30 en la isla, discurso en el cual una élite descendiente de la clase criolla bajo la colonización española estableció las dinámicas raciales, culturales y de identidad en Puerto Rico para afianzar su poder ante la colonización estadounidense. Con esto en mente, mi propósito en este trabajo es analizar "El entierro de Chianita: un complot chino" de Montero vis-à-vis el ensayo El prejuicio racial en Puerto Rico de Tomás Blanco con el fin de subrayar cómo el discurso del 30 pervive en la actualidad y, entre otros asuntos, escamotea proyectos antirracistas. ${ }^{4}$ La primera parte de este trabajo analizará los dos ejemplos que Montero incluye en su ensayo para justificar la presencia de personajes como Chianita. ${ }^{5}$ La segunda parte estudiará las conexiones entre el ensayo de Blanco y el de Montero y mencionará posibles estrategias que ayuden a combatir el racismo institucionalizado.

Cabe hacer algunas salvedades antes de proseguir con este análisis. En primer lugar, queda fuera del alcance de este trabajo una crítica de las descripciones que Montero hace de los manifestantes y de su argumento sobre cómo éstos no enterraron a otros personajes de la televisión puertorriqueña que pueden ser un tanto ofensivos. No he reparado en estos asuntos gracias a que ya han sido discutidos por otras personas. ${ }^{6}$ En segundo lugar, entiendo que parte de la propuesta principal de Montero tiene que ver con la censura, es decir, con cierta preocupación ante la posibilidad de que cualquier protesta impida la libre expresión artística. El problema estriba en que regañar y tildar de terroristas a los que protestan pacíficamente contribuye, de una forma u otra, a criminalizar el derecho a protestar. Por ende, la lógica que Montero emplea indirectamente sirve para restringir la libertad de expresión que ella busca defender. Como ella señala, Univisión no tenía que "ceder" ante las presiones de los activistas y si lo hizo, dudo que lo hiciera ante el temor de que se llevara a cabo un acto terrorista. De Univisión no haber cedido, los manifestantes hubieran buscado otras formas de protestar y difundir sus acertadas premisas en cuanto a lo ofensivo que resulta que un artista blanco se oscurezca el rostro para representar a un personaje negro.

Como colofón, ya que he mencionado la censura, deseo afirmar que no apoyo la eliminación de la columna de Montero ni boicots a sus libros como algunos han propuesto. Aunque me parece que en algunos casos la representación de personajes afrodescendientes es estereotípica, su obra literaria es sumamente valiosa y en un sinnúmero de sus columnas ensayísticas Montero ha analizado lúcidamente los males sociales y políticos que tanto aquejan a la isla.7 Este estudio que hago va más allá de Montero. Lo que intento reiterar es que el que actualmente en textos como el de ella haya puntos en común con un texto de Blanco lo que demuestra es que las propuestas raciales de la Generación del 30-las cuales, como mencioné, tienen vínculos con las de la élite criolla durante la colonización española-se han incrustado de tal manera que resurgen constantemente y tienen consecuencias muy reales para los afrodescendientes que son discriminados y microagredidos.

\section{Dos ejemplos: la ópera y el teatro bufo cubano}

Uno de los aspectos que más críticas suscitó fue el que Montero tildara a los manifestantes de terroristas. Esto ha sido refutado por varios intelectuales y no repararé exhaustivamente en el asunto. ${ }^{8}$ Sin embargo, sí deseo aludir al empleo de una retórica orientalista en el texto en cuestión. No hay un terrorismo "benigno" o "aceptable" y otro que no lo es, pero nótese que los ejemplos de Montero remiten a una religión y a una región en particular. Su incomodidad por la "otomanización" del país a causa de la proyección de telenovelas turcas así como la alusión a que los activistas antirracistas se asemejan al ejército de Mao y a los terroristas que atacaron a Charlie Hebdo en Francia fomentan la creencia de que los terroristas son musulmanes y/o asiáticos ya que no se alude a terroristas de otros contextos como, por ejemplo, el irlandés, el vasco y el griego. Esto es problemático porque forma parte de proposiciones que son "lax and characteristically Orientalist...-Muslims are enraged 
at modernity, Islam never made the separation between church and state, and so on and so on-all of them pronounced with an extreme level of generalization and with scarcely a mention of the differences between individual Muslims, between Muslim societies, or between Muslims traditions and eras" (Said, Orientalism 341). Sutilmente esto contribuye al miedo que muchos tienen hacia "lo oriental" debido a que, supuestamente, dicho grupo pretende atacar a los demás: "The perfidious Chinese, half-naked Indians, and passive Muslims are described as vultures for 'our' largesse and are damned when 'we lose them' to...their unregenerate Oriental instincts: the difference is scarcely significant. These contemporary Orientalist attitudes flood the press and the popular mind. Arabs, for example, are thought of as...terroristic...lechers...” (Said, Orientalist 108). En suma, aunque Montero probablemente usó estas comparaciones de modo sarcástico e hiperbólico, lamentablemente éstas perpetúan la retórica orientalista que homogeniza a los chinos y musulmanes y los considera personas violentas. ${ }^{9}$

Ahora bien, para justificar la presencia de personajes como Chianita, Montero recurre a ejemplos de la ópera entre los cuales resalta el Otello de Verdi puesto que el tenor que interpreta este papel tradicionalmente se oscurece el rostro para acercarse al imaginario que predomina sobre cómo debe verse un moro. La autora enfatiza que algunos de los Otellos más "deslumbrantes" han sido los interpretados por Plácido Domingo. En tono sarcástico alude a que como los activistas están "puestos a enterrar, por favor, que esperen a la próxima temporada del Met, vayan a la representación de Otello-si es con el ruso Aleksandrs Antonenko, mejor-,y allí, de paso, junto con Otello y Antonenko, entierren a Plácido Domingo". Su sugerencia se presta para dos lecturas. Por un lado, parecería que los activistas deben enterrar a Otello, a Antonenko y a Domingo. Por otro lado, otra posible lectura implica que Otello y Antonenko se les unan a los activistas para enterrar a Domingo. La anfibología que permite la participación de Otello y Antonenko en el entierro de Domingo es sugerente porque desde el 2015 el Met ha descartado el uso de "blackface" para los tenores que hacen el papel de Otello. En otras palabras, el latvio Antonenko hace el papel de moro sin teñirse la cara mientras que por años Domingo sí se oscureció el rostro para actuar de Otello. Por lo tanto, una lectura implicaría que los activistas tal vez no objetarían a la nueva política del Met y por consiguiente solamente Domingo amerita ser enterrado. La otra lectura de este planteamiento señalaría que hay que enterrar al personaje de por sí y a todos los que han interpretado este papel sin importar si se han pintado el rostro o no.

Tras aludir al caso de Otello, Montero entonces cuestiona qué le depara a una soprano como la letona Kristine Opolais quien se maquilla para parecer japonesa e interpretar a Madame Butterfly. Por último, la autora apunta a que hay un tipo de terrorismo detrás de estos entierros simbólicos que ella compara al de los talibanes: "no dudaría que un comando talibán se presente en la Ópera de Milán, y entierre (de verdad, a bombazo limpio) a los actores y el público que aplaude Otello, ya que se burlan de un musulmán atormentado por los celos".

Hay par de aspectos de este ejemplo que hay que rebatir o al menos cuestionar. Por un lado, la decisión del Met de rechazar que los tenores blancos se pinten la cara de negro no ha sido mal vista. El New York Times entrevistó a Barlett Sher, el encargado de la producción de Otello, y a Francesca Zambello, la directora artística de la Opera Nacional de Washington. Ambos explicaron que el "blackface" es una tradición que hoy día es innecesaria y que poco a poco su aparición en la ópera está siendo superada en su totalidad. Zambello va más allá y arguye que de tener que contratar a un tenor para el papel de Otello trataría de encontrar a un tenor negro, pero, que de no hallarlo, contrataría a uno blanco sin proceder a oscurecerle el rostro. El periódico en cuestión también obtuvo declaraciones de Plácido Domingo quien explicó que en su momento personificó al personaje con la cara oscurecida con el propósito de aumentar la verosimilitud del mismo. Sin embargo, el tenor expresó que le encantaría ver la nueva producción con Antonenko interpretando a un Otello sin el rostro oscurecido (Domingo citado en Cooper). Cabe entonces preguntarse por qué Montero usa este ejemplo si, para empezar, a Domingo-el tenor que hizo las personificaciones de Otello más famosas de los ochenta y noventa-no parece molestarle el cambio llevado a cabo por el Met ni que Antonenko interprete el papel sin maquillaje oscuro.

Con todo, hay otro asunto que hay que analizar y es que muchas obras del canon operístico-así como de los cánones de otros tipos de arte-tienen vínculos con la ideología imperialista y, por lo tanto, la aparición de personajes no europeos debe estudiarse minuciosamente para ver qué dicen estos textos sobre las dinámicas raciales y étnicas en un contexto imperial. Me refiero a que-como señala Said en su estudio de Aida de Verdi-este arte

can be enjoyed and interpreted as a kind of curatorial art, whose rigor and unbending frame recall, with relentlessly mortuary logic, a precise historical moment and a specifically dated aesthetic form, an imperial spectacle designated to alienate and impress an almost exclusively European audience. Of course, this is very far from Aida's position in the cultural repertory today. And certainly it is true that many great aesthetic objects of empire are remembered and admired without the baggage of domination that they carried through the process from gestation to production. Yet the empire remains, in inflection and traces, to be read, seen, and heard. (Culture and Imperialism 130) ${ }^{10}$

Esto no implica que el tipo de arte que forma parte de estas dinámicas imperiales-en este caso la ópera-deba 
prohibirse o dejarse de estudiar o que se deba dejar de tocar la "Marcha triunfal" de Aida en graduaciones como es costumbre en muchas instituciones escolares y universitarias. Tampoco implica que dicho arte sea de mala calidad. Concuerdo con Said cuando aclara que "by looking at culture and imperialism carefully we may discern various forms in the relationship and we shall see that we can profitably draw connections that enrich and sharpen our reading of major cultural texts. The paradoxical point, of course, is that European culture was no less complex, rich, or interesting for having supported most aspects of the imperial experience" (Culture and Imperialism 163).

A lo que me refiero es a que si algunas óperas están vinculadas a contextos imperiales, una lectura cuidadosa debe llevar a ver la problemática del uso de "blackface" para un público imperial. Así pues, el ejemplo de Montero subraya la resistencia a dejar a un lado tradiciones que surgen de situaciones coloniales donde se crea a un otro racializado para entretener e ignora lecturas críticas que no buscan restarle valor artístico a la ópera sino ver sus conexiones con ideologías imperialistas con el fin de desarrollar nuevas lecturas artísticas que saquen dichas representaciones del plano caricaturesco que el consumo pasivo y acrítico fomenta. ${ }^{11}$

El otro ejemplo que Montero usa para justificar la presencia de personajes como Chianita es el teatro bufo cubano: "En Cuba sería impensable que, al revivir una obra del teatro bufo - y se reviven a menudo: con el negrito, el gallego y la mulata - se presentaran unos 'manifestantes' a enterrarlos". En este tipo de obra teatral, lo común es que un actor blanco se pinte el rostro de negro para hacer el papel del negrito, toda vez que en ocasiones una actriz blanca se pinta la cara para representar a la mulata.

Montero no dialoga con la crítica que se le ha hecho a estas representaciones teatrales en Cuba. Por ejemplo, en su estudio "El negrito y la mulata en el vórtice de la nacionalidad" Inés Martiatu Terry ha demostrado lúcidamente cómo el origen del teatro bufo decimonónico tiene conexión con el teatro del Siglo de Oro en España. Su análisis evidencia cómo desde el siglo XVII en el teatro se viene construyendo al negro como un sujeto racializado y subalterno a partir del discurso hegemónico de la blancura (280). En resumidas cuentas, la práctica del "blackface" en el teatro bufo cubano surge del contexto colonial donde la esclavitud era aceptable. Luego, en el siglo XIX-en el contexto de las guerras de independencia-se representa al negro como ese otro que no debe ser incluido dentro del proyecto de nación (Martiatu Terry 297; Rivero 32). Por ende, Martiatu Terry enfatiza que

la aceptación y persistencia de estos esquemas y de estas operaciones de distorsión y enmascaramiento demuestran cómo la falsedad de los modelos no impide que sean aceptados y que perseveren en el imaginario del pueblo hasta nuestros días. De ahí la importancia de deconstruirlos y de ofrecer una alternativa teórica que les restituya su identidad verdadera...[puesto que] estos personajes en el teatro bufo son un claro ejemplo de suplantación de la fea cara de la explotación esclavista y racista. (Martiatu Terry 292)

El estudio de Martiatu Terry es un claro ejemplo de cómo en Cuba se ha puesto en tela de juicio los aspectos supuestamente admirables del teatro bufo aun cuando Montero recalque que figuras como Alejo Carpentier han defendido este tipo de representación artística.

Si bien no puedo afirmar ni negar la premisa de Montero respecto a lo impensable que sería una protesta en Cuba a raíz de la puesta en escena de una obra de teatro bufo, sí cuestiono la implicación que se desprende de su ensayo acerca del supuesto consumo pasivo y acrítico de este tipo de obra teatral en suelo cubano. ${ }^{12}$ Esto me lleva al asunto que realmente deseo reiterar: este tipo de crítica que se le ha hecho al teatro bufo no podría descartarseasí, sin más-por ser una importación de ideas estadounidenses. Aunque sí hubo influencia de los minstrels en el teatro bufo cubano (Martiatu Terry 282; Rivero 33-34), la crítica que se le ha hecho al teatro no se desprende de lo que haya ocurrido o no en Estados Unidos dado que la misma parte de la reprobación al legado colonial español y a los parámetros raciales que se usaron para implementar y justificar la esclavitud. En otras palabras, la crítica que en Cuba se le ha hecho al "blackface" demuestra que éste tiene vínculos con el colonialismo y ha afianzado actitudes racistas a través del arte dramático.

\section{“Importaciones" y "sesgos colonizados": Mayra Montero vis-à-vis Tomás Blanco}

Mi análisis de los ejemplos de Montero demuestra que éstos no sólo tienen vínculos con el pasado colonial sino que actualmente tanto en la ópera como en Cuba la práctica de pintarse la cara de negro o está cayendo en desuso o ha sido criticada. No obstante, una de las particularidades del ensayo de Montero es que la crítica hacia Chianita es interpretada como algo foráneo que no tiene lugar o lógica alguna en Puerto Rico. Quiero indagar en este aspecto del ensayo de Montero, no sin antes repasar las propuestas de Tomás Blanco acerca de lo racial. ${ }^{13}$

Recordemos que Blanco fue parte de la llamada Generación del 30 en Puerto Rico, un grupo que ante la convocatoria que lanzó la Revista Índice en 1929 escribió una serie de ensayos para explicar cómo son los puertorriqueños y qué le depara a la isla a unos treinta años de la invasión estadounidense de 1898. Si bien el ensayo más conocido de Blanco es Prontuario histórico de Puerto Rico (1935), es El prejuicio racial en Puerto Rico (1937) el que repercute en el ensayo de Montero. 
Blanco y otros intelectuales de la década del treinta procuraban establecer una identidad nacional distinta a la estadounidense. A simple vista, esta forma de pensar parece positiva puesto que pretende desligarse de lo estadounidense en aras de afianzar proyectos autóctonos. Con todo, al analizar los postulados de Blanco vemos que algunos son problemáticos y racistas.

La premisa de Blanco en El prejuicio se puede resumir de la siguiente manera: en Puerto Rico no hay prejuicio racial porque a lo largo de su historia las relaciones entre blancos y negros-desde la época de la esclavitud hasta el presentehan sido "benignas" (Blanco 12,14, 23-24, 43), toda vez que en la isla nunca hubo muchos esclavos (Blanco 18-20). La esclavitud en Puerto Rico fue "light" (Blanco 23-24, 28-29) y permitió no sólo la mezcla racial (Blanco $26,35-36,48$ ) sino la civilización de los negros al éstos aceptar las costumbres hispanas. Consiguientemente, la cantidad de negros "puros" en el país no es muy alta debido a la mezcla: "Nuestro pueblo tiene abundante sangre negra, aunque, en general, casi no existen negros puros, y aunque nuestra población de color está completamente hispanizada culturalmente y son muy escasas las aportaciones africanas a nuestro ambiente, salvo en el folklore musical" (Blanco 51). Así pues, las relaciones entre blancos y negros supuestamente se caracterizan por la convivencia.

La idea de una esclavitud benigna es insostenible y obvia las sublevaciones que los esclavos llevaron a cabo. ${ }^{14}$ Como bien resume Díaz Quiñones, Blanco "congela en una figura inmóvil los procesos históricos. Suprime la historia de los impugnadores del régimen esclavista y colonial, y el discurso de los rebeldes. Suprime también...la complejidad y la opresión del mundo esclavista, y el rencor que generó el racismo que hizo posible la esclavitud" (19).

Por otro lado, la mezcla racial que presenta Blanco así como la civilización hispánica de los afrodescendientes a la que alude lleva a un blanqueamiento cultural y fenotípico que-en el contexto de la Generación del 30-ataca la racialización que el discurso estadounidense utilizó para justificar la colonización de Puerto Rico. Me refiero a que el discurso imperial estadounidense vio a los puertorriqueños como bárbaros, atrasados, infantilizados y negros que necesitaban un protector civilizado, paternalista y blanco que los salvara y los dirigiera (Alamo-Pastrana 6). Con todo, las premisas de Blanco no dejan de tener una ideología racista que escamotea lo afrodescendiente y en el fondo favorece lo hispano y lo percibido como blanco. ${ }^{15}$

Ahora bien, las afirmaciones de Blanco con respecto a la esclavitud, las relaciones entre blancos y negros y la alegada ausencia de prejuicio racial en la isla parten de la comparación que él hace entre Puerto Rico y Estados Unidos: "Comparado con las más intensas explosiones de virulencia, nuestro prejuicio es un inocente juego de niños" (Blanco 4). Por eso el autor que nos ocupa reitera que en la isla nunca hubo leyes como las "Jim Crow" ni linchamientos (Blanco
31,35). De esta forma Blanco pretende demostrar que Puerto Rico es un país civilizado que carece de los conflictos raciales que hay en Estados Unidos. Según este argumento, Puerto Rico-al menos desde un punto de vista moral-es superior a Estados Unidos (Díaz Quiñones 40; Godreau 26).

La comparación que Blanco hace entre Puerto Rico y Estados Unidos lo lleva a concluir que en la isla no hay prejuicio racial. Por lo tanto, cuando alguien se queja de prejuicio racial es a fuerza de: 1-casos discriminatorios llevados a cabo por individuos racistas o 2 -discursos raciales estadounidenses impuestos al contexto puertorriqueño. Los casos discriminatorios son aislados y no son motivo de preocupación puesto que-según Blanco-los paisanos no racistas se encargan de darles una reprimenda a los que sí lo son (Blanco 36-40). Los problemas surgen cuando se "importan" e imponen discursos raciales foráneos:

...el cretinismo espiritual de ciertos criollos tiende a exagerar la existencia, el grado y la calidad o esencia de nuestro prejuicio...[M]ás que nada contribuye a ello la presencia importada, con algunos ciudadanos continentales, del rigor del prejuicio norteamericano, que siempre se adivina aunque no se manifieste muchas veces. Se produce entonces en algunos isleños una imitación poco convincente de los ajenos vicios...En el fondo no es más que un tácito sometimiento intelectual a los juicios extraños, una pueril e ineficaz reacción defensiva de colonialismo mental. (Blanco 60-61, énfasis mío)

Comparar a Puerto Rico con Estados Unidos lo que ha hecho es obviar que ya en la isla había prejuicio racial que surge del legado colonial y esclavista español (AlamoPastrana 8). Mediante esta comparación, Blanco ignora la ideología racista que viene de la colonización previa de Puerto Rico por parte de España y se circunscribe a las dinámicas de la colonialidad del poder que han marcado la pauta de las interacciones sociales y económicas desde la conquista hasta el presente (Quijano 183).

Este somero análisis de El prejuicio racial en Puerto Rico es necesario debido a las similitudes entre algunas de sus propuestas y las de "El entierro de Chianita: un complot chino." Obviamente el ensayo de Blanco-de unas ochenta páginas-abarca más temas que el ensayo de Montero que se circunscribe a su columna periodística. Además, Montero, a diferencia de Blanco, no estipula a rajatabla que en Puerto Rico no hay racismo. No obstante, el punto que tienen en común es la manera en la que ambos arguyen que esos que protestan o se quejan de alguna forma a causa de prácticas racistas, han importado o copiado ideas estadounidenses que no tienen relevancia en la isla. Por ende, Montero les reitera a los lectores de su ensayo lo siguiente acerca de los que enterraron a Chianita: "Nótese que son actitudes importadas de los Estados Unidos, hasta lo dijeron en inglés, "blackface", vean el sesgo del colonizado" (énfasis mío). Remito a estas aseveraciones de Blanco porque es en ellas donde se ve la conexión con el ensayo de Montero: 
"contribuye [a exagerar de la existencia, el grado y la calidad o esencia del prejuicio racial] la presencia importada, con algunos ciudadanos continentales, del rigor del prejuicio norteamericano...En el fondo no es más que un tácito sometimiento intelectual a los juicios extraños, una pueril e ineficaz reacción defensiva de colonialismo mental" (Blanco 6o-61, énfasis mío).

Por un lado, asumir que los casos de prejuicio racial que hay en la isla son creados, imaginados o exagerados a partir de la importación de parámetros raciales de Estados Unidos impide que se pueda tener una discusión seria acerca de los problemas raciales ya que se alude a que estos no son reales dado que son foráneos. Por otro lado, el vínculo entre Montero y Blanco apunta a que entre algunos intelectuales los debates en torno a lo racial no han cambiado mucho en ochenta años, toda vez que los discursos de la llamada Generación del 30 siguen vigentes en el país. En otras palabras, la conexión que describo entre el texto de Blanco y el de Montero es un ejemplo de cómo el discurso racial del treinta se institucionalizó en la isla. Esto ha contribuido a un silenciamiento en torno a lo racial que afianza "the pervasive myths of racial democracy and social and intelectual mores [that] demand that Afro-Puerto Ricans not speak from a position of discontent even in the face of racist assaults" (Roy-Féquière 258).

\section{Derroteros y conclusiones}

He analizado el modo problemático en que se usan ejemplos de la ópera y del teatro bufo cubano para justificar la existencia de personajes como Chianita en el Puerto Rico del siglo XXI. Asimismo, he subrayado la manera en que desde la década del treinta en ciertos núcleos boricuas se critica a los que señalan las prácticas racistas que hay en la isla. Esto se debe-entre otros factores-a que se establece una comparación entre las dinámicas raciales estadounidenses y las puertorriqueñas en la cual las puertorriqueñas son "benignas." En todo caso, los incidentes de discrimen son aislados y los que insisten en que hay racismo en la isla lo hacen porque han importado creencias estadounidenses. Esa importación los convierte-según las premisas de Blanco y, hasta cierto punto, las de Montero-en colonizados cuyas propuestas a fin de cuentas lo que hacen es dividir al país o impedir que la gente trabaje (Montero). Sostengo que estos planteamientos no hacen más que impedir que se lleven a cabo conversaciones serias que aludan al racismo y a la búsqueda de formas para erradicarlo hasta donde sea posible. A modo de conclusión, deseo aludir a algunos derroteros para continuar tan importante diálogo.

Primeramente, es necesario retomar el estudio del ensayo de interpretación cultural no solo en el plano de la literatura puertorriqueña sino en el plano de la literatura latinoamericana. Concuerdo con Joy Landeira cuando explica que "a diferencia de mediados de siglo XX, cuando se enseñaba el ensayo como uno de los cuatro géneros reconocidos, al lado de la narrativa, la poesía y el teatro, hoy en día numerosos críticos no lo admiten, y hay muchos programas universitarios que sólo lo enseñan en sus clases de composición básica...y no como manera de...observar las transformaciones culturales" (559). No deseo implicar que esta práctica se ha extendido a todas las instituciones de enseñanza secundaria y universitaria. Sin embargo, los planteamientos que enfatizan que los cursos del ensayo latinoamericano no son necesarios porque el contenido puede ir "a caballo" en los cursos de literatura decimonónica y contemporánea que se enfocan en la narrativa ignoran que ha sido en la ensayística latinoamericana donde se han planteado proyectos relacionados a lo racial, proyectos que en algunos casos han tenido consecuencias muy reales sobre las dinámicas raciales en sus respectivos contextos culturales. Basta con mencionar Casa grande e senzala de Gilbeto Freyre y Contrapunteo cubano del tabaco y el azúcar de Fernando Ortiz y cómo se han usado para asumir-erróneamente-que en Brasil y en Cuba hay democracias raciales. Otros ejemplos fundamentales son $L a$ isla al revés de Joaquín Balaguer y La política de Trujillo de Manuel Arturo Peña Batlle, ensayos que en el contexto dominicano han servido para afianzar un discurso nacional afrófobo y antihaitiano. En suma, el estudio del ensayo es crucial para las discusiones en torno a lo racial puesto que permite analizar cómo lo planteado en algunos de estos textos repercute hoy día.

En segundo lugar, hay que buscar enfoques que rebasen la comparación tradicional que se ha hecho entre Estados Unidos y Puerto Rico a la hora de hablar de lo racial. Como mencioné previamente, dicho modelo comparativo-entre otros asuntos-escamotea el racismo que ya existía en la isla por culpa de la colonización española dado que, la comparación de las dinámicas raciales estadounidenses y las puertorriqueñas, permite que algunos concluyan que en Puerto Rico no hay discrimen racial. Un punto de partida para salir de este impasse es el de imbricación racial que propone Carlos Alamo-Pastrana en su estudio Seams of Empire: "As a methodological concept, racial imbrication directs scholars to the unexpected yet organized points of overlap among seemingly diverse points of difference" (12). Esto permite superar "the failed method of comparison in the study of race" debido a que "[it] opens up the possibilities for thinking about diaspora and race outside a conservative and minority nationalisms that erase the work, ideas, and contributions of marginalized groups" (149-150). Asimismo, pensar el Caribe en términos culturales en vez de geográficos ayudaría a ver aspectos comunes que tienen los lugares donde imperó la cultura de la plantación (i.e. desde el sur de los Estados Unidos hasta Brasil). ${ }^{16}$ 
El estudio del ensayo de identidad nacional y/o cultural así como la necesidad de rebasar los modelos comparativos para estudiar las dinámicas raciales son algunos derroteros para acercarse de manera constructiva a los problemas raciales en Puerto Rico. Deseo añadir algo más: la necesidad de dejar a un lado proyectos que anclen las identidades puertorriqueñas en un imperativo geográfico $\mathrm{y}$ - por endesilencien a los puertorriqueños que residen o han residido en Estados Unidos. Si para Blanco en los treinta y para Montero en la actualidad hay importaciones de ideas estadounidenses, entonces habría que tomar en cuenta no solo la "importación" de éstas por parte de los que siempre han vivido en Puerto Rico sino las que llegan a través de los que residen o han residido en Estados Unidos. Ponerse a buscar los nombres de cada uno de los participantes de la protesta en contra de Chianita para determinar si han residido en los Estados Unidos es fútil. Lo que quiero apuntar es que las premisas de que las protestas hacia personajes como Chianita o las críticas hacia el racismo en la isla parten de importaciones por personas cuyas mentes son colonizadas indirectamente atacan a la diáspora puertorriqueña y a sus remesas culturales que contribuyen a que se cuestionen y se derrumben jerarquías raciales, sexuales, étnicas y de género. ${ }^{17}$ La diáspora ha influido en el panorama cultural y político puertorriqueño (Flores 47, 144). Insinuar que estas remesas culturales no tienen cabida en suelo boricua demuestra un sesgo o "punto ciego" entre los que defienden un proyecto nacional que, al intentar dar al traste con lo estadounidense, no admite las influencias de la diáspora.

En resumen, el ensayo de Montero publicado a raíz de la protesta en contra de Chianita apunta a cómo el discurso racial de la generación del treinta así como sus estrategias discursivas para atacar a los que protestan ante el discrimen racial en la isla sigue vigente. Este aspecto me ha llevado a ver las conexiones entre su ensayo y El prejuicio racial en Puerto Rico de Tomás Blanco y a problematizar algunos de los ejemplos que Montero presenta (i.e. ópera, teatro bufo) para justificar su regaño hacia los manifestantes.

Mi trabajo aboga por más estudios del ensayo, modelos que superen el impasse de las comparaciones de las dinámicas raciales entre EEUU y Puerto Rico y la apertura hacia las remesas culturales. Estos enfoques no son excluyentes ni exhaustivos. Sin embargo, éstos son útiles para revisar proyectos culturales y políticos con el fin de propiciar lecturas que aprecien la diversidad y combatan el racismo en el Puerto Rico contemporáneo.

\section{NOTAS}

1 Puerto Rico se encuentra en quiebra tras un período de recesión que empezó a finales de 2005 . Actualmente hay una junta de control fiscal -impuesta por Estados Unidos-que decide cómo se manejan las finanzas locales. La junta privilegia el pago de la deuda y lo que se les debe a los bonistas y ha implementado medidas austeras que afectan negativamente a la clase trabajadora. Las tasas de desempleo y criminalidad son altas. Este era el panorama antes del azote del huracán María en septiembre de 2017. Dicho azote ha exacerbado la crisis en la cual Puerto Rico ya se encontraba.

2 El comentario de Meyer resulta contradictorio puesto que del Villard y Richardson eran-precisamenteactrices negras.

3 El comentario que Meyer escribió el 2 de noviembre de 2016 en su cuenta de Facebook fue el siguiente: "Me acaba de llamar Primera Hora para que opine sobre la marcha en contra de Chianita! Bendito, la hicieron famosa otra vez y hace ya días que se decidió que no iba esa pequeña participación. No vale la pena herir o molestar a nadie por cantar Chianita Gobernadora. Le han deseado a mi amada Chana la muerte y realmente es en lo único que no los puedo complacer, porque cómo sacas a Chianita del corazón del pueblo?”
4 Para indagar en cómo el discurso racial de la Generación del 30 sigue vigente en Puerto Rico, ver Scripts of Blackness: Race, Cultural Nationalism, and U.S. Colonialism in Puerto Rico de Isar Godreau y "Absorber, engullir y diluir: blanqueamiento y mestizaje problemático en la ensayística de Emilio S. Belaval" de Violeta Lorenzo.

5 Esta fuera del alcance de este trabajo analizar las particularidades del uso de "blackface" en la televisión puertorriqueña y los pormenores de la disputa entre Meyer y del Villard en los setenta ya que esto ha sido estudiado por Rivero en su libro Tuning Out Blackness.

6 Para más información ver la respuesta que SantosFebres publicó el 6 de noviembre de 2016 por Facebook. Ver también las respuestas de Hilda Lloréns, Harry Franqui-Rivera, William García, Bárbara Abadía Rexach e Isar Godreau así como los comentarios del activista Weldo Romero Joseph en el artículo de Brenda Peña López que fue publicado el 2 de noviembre de 2016 en el periódico El Nuevo Día.

7 En La última noche que pasé contigo el personaje Celia desea ser negra porque entiende que las mujeres negras están sexualmente satisfechas. En esa misma novela se alude al cliché del tamaño enorme de los genitales de los negros y a su sexualidad irrefrenable. Ejemplo de esto es el botero con quien Celia tiene un amorío y 
que es descrito como un monstruo marino con un gran miembro viril (Montero, La última noche 151-154). Si bien en esta novela erótica se presenta la exploración sexual como fuente de conocimiento, en ésta se repiten ciertos mitos sobre la sexualidad de los negros. Para información preliminar sobre el mito de la sexualidad negra ver, de Santos-Febres, "El color de la seducción".

8 Franqui-Rivera aclara que aunque el semanario Charlie Hebdo sí ha tenido posturas racistas, la comunidad internacional denunció el ataque violento que sufrió. Es decir, protestar en contra del racismo no equivale a justificar la violencia y el terrorismo, aspecto que en ningún momento los activistas en Puerto Rico hicieron. Asimismo, Santos-Febres explica que las comparaciones de Montero son exageradas y desacertadas y carecen de contextualización histórica.

9 Por otro lado, en otros contextos Montero ha escrito positivamente sobre la influencia china en el Caribe. Ver su ensayo "Here Comes the Chinaman: Another Song of Cuban Identity" y su novela Como un mensajero tuyo.

10 La cantidad de estudios críticos de corte postcolonial sobre el Otello de Shakespeare, el de Verdi y otras composiciones musicales y teatrales es vasta. Las particularidades de los mismos están fuera del alcance de este trabajo. Sin embargo, estos textos pueden servir como punto de partida para acercarse al tema: PostColonial Shakespears (Loomba y Orkin, eds.), Blackness in Opera (Andre, Bryan, Saylor, eds.) y Orientalism and Representations of Music in the Nineteenth-Century British Popular Arts (Mabilat).

11 Said comenta algo similar a lo que planteó en el capítulo "The Empire at Work: Verdi's Aida" de su libro Culture and Imperialism.
12 Montero no explica las razones por las cuales este tipo de protesta sería impensable en Cuba. ¿Es impensable porque realmente son muy pocos los que critican este tipo de teatro y no hay cuórum para una protesta? ¿O será porque el sistema político de Cuba restringe el derecho a protestar?

13 Para más información sobre la influencia de Tomás Blanco en los discursos raciales puertorriqueños ver “Tomás Blanco: racismo, historia, esclavitud” de Arcadio Díaz Quiñones y Scripts of Blackness: Race, Cultural Nationalism, and U.S. Colonialism in Puerto Rico de Isar Godreau.

14 Varios investigadores han demostrado que, por un lado, hubo sublevaciones de esclavos en Puerto Rico y, por otro lado, no todos los afrodescendientes fueron esclavos. Ver Esclavos rebeldes de Guillermo Baralt para más información.

15 Ver los libros de Isar Godreau y Magali Roy-Féquière citados en este trabajo para más información sobre el blanqueamiento detrás de las premisas de Blanco. El estudio de Díaz Quiñones también tiene información al respecto.

16 Para más información sobre las distintas maneras de definir el Caribe, ver "La invención del Caribe desde 1898 (Las definiciones del Caribe como problema histórico, geopolítico y metodológico)." En este artículo, Gaztambide aboga por una definición cultural que permita ver los aspectos comunes que tienen lugares como el sur de los Estados Unidos y el Caribe.

17 Por remesas culturales me refiero a "cultural customs and practices, ideological orientations, forms of artistic expression, and ideas of group identity acquired in diaspora settings [that] are remitted to homeland societies" (Flores 44).

\section{OBRAS CITADAS}

Abadía-Rexach, Bárbara I. "El duelo del racismo.” El Nuevo Día, 7 Nov. 2016, www.elnuevodia.com/opinion/ columnas/elduelodelracismo-columna-2259692/.

Alamo-Pastrana, Carlos. Seams of Empire: Race and Radicalism in Puerto Rico and the United States. University Press of Florida, 2016.

André, Naomi Adele., et al., editors. Blackness in Opera. University of Illinois Press, 2012.

Baralt, Guillermo A. Esclavos rebeldes: conspiraciones y sublevaciones de esclavos en Puerto Rico (1795-1873). Ediciones Huracán, 2006.

Blanco, Tomás. El prejuicio racial en Puerto Rico. Biblioteca de Autores Puertorriqueños, 1948.

Cooper, Michael. “An ‘Otello’ Without Blackface Highlights an Enduring Tradition in Opera." New York Times, 17 Sept.
2015, www.nytimes.com/2015/09/20/arts/music/an-otello-without-the-blackface-nods-to-modern-tastes.html.

Díaz Quiñones, Arcadio. “Tomás Blanco: racismo, historia, esclavitud”. Introducción. El prejuicio racial en Puerto Rico. Por Tomás Blanco. 3ra ed. Río Piedras: Huracán, 1985. 13-91.

Flores, Juan. The Diaspora Strikes Back: Caribeño Tales of Learning and Turning. Routledge, 2009.

Franqui-Rivera, Harry. "Mayra Montero denuncia como gringada las demonstraciones en contra de Black faces en Puerto Rican T.V.” In Coherent Thoughts, WordPress, 6 Nov. 2016, incoherentthoughtsblog.com/2016/11/06/ mayra-santos-denuncia-como-gringada-las-demonstraciones-en-contra-de-black-faces-en-puerto-rican-t-v/. 
García, William. "The Burial of Chianita La Negra: White Fragility and Blackface in Puerto Rico." Latino Rebels, 8 Nov. 2016, www.latinorebels.com/2016/11/o8/the-burial-of-chianita-la-negra-white-fragility-and-blackface-in-puerto-rico/.

Gaztambide-Géigel, Antonio. "La invención del Caribe a partir de 1898 (las definiciones del Caribe como problema histórico, geopolítico y metodológico.” Tan lejos de Dios...Ensayos sobre las relaciones del Caribe con Estados Unidos, por Antonio Gaztambide-Géigel, Ediciones Callejón, 2006, pp. 23-58.

Godreau, Isar. "Respuesta a la acusación de "censura” en torno a la no-invitación de Mayra Montero para dictar una conferencia magistral.” Facebook. 6 Dec.2016. www. facebook.com/notes/rima-brusi-gil-de-lamadrid/respuesta-a-la-acusaci\%C3\%B3n-de-censura-en-torno-a-la-noinvitaci\%C3\%B3n-de-mayra montero/10157867606610187/

---. Scripts of Blackness: Race, Cultural Nationalism, and U.S. Colonialism in Puerto Rico. University of Illinois Press, 2015.

Landeira, Joy. “Continuidades y diferencias ensayísticas en My Own Private Cuba: Essays on Cuban Literature and Culture de Gustavo Pérez Firmat." Revista Iberoaméricana, vol. LXXVIII, no. 240, 2012, pp. 555-66.

Lloréns, Hilda. "Una breve reflexión sobre la práctica blanca de pintarse el rostro de negro/negra (o "rostro-negro”/blackface) en Puerto Rico.” Createliveflourish, 6 Nov. 2016, createliveflourish.blogspot.com/2016/11/ una-breve-reflexion-sobre-la-practica_6.html?m=1.

Loomba, Ania, and Martin Orkin, editors. Post-Colonial Shakespeares. Routledge, 2011.

Lorenzo, Violeta. “Absorber, engullir y diluir: blanqueamiento y mestizaje problemático en la ensayística de Emilio S. Belaval." Chasqui, vol. 46, no. 2, 2017, pp. 256-73. Mabilat, Claire. Orientalism and Representations of Music in the Nineteenth-Century British Popular Arts. Routledge, 2017.
Martiatu Terry, Inés María. "El negrito y la mulata en el vórtice de la nacionalidad." Afrocubanas: historia, pensamiento y prácticas culturales, edited by Daisy Rubiera Castillo, et.al., Editorial de Ciencias Sociales, 2011, pp. 273-98.

Meyer, Ángela. No vale la pena molestar a nadie. Facebook. 2 Nov. 2016. www.facebook.com/angela.meyer.942?fref=searchMontero, Mayra. "El entierro de Chianita: un complot chino.” El Nuevo Día, 5 Nov. 2016, www.elnuevodia.com/opinion/columnas/elentierrodechianitauncomplotchino-columna 2259286/.

---. "Here Comes the Chinaman: Another Song of Cuban Identity.” Trad. Vivian Otero. Sargasso, no. 1, 20062007, pp. 1-8.

---. La última noche que pasé contigo. Tusquets, 1994.

Peña López, Brenda I. "Sepultan a Chianita por considerarla racista.” El Nuevo Día, 3 Nov. 2016, www.elnuevodia. com/entretenimiento/tv/nota/sepultanachianitaporconsiderarlaracista 2258196/.

Quijano, Aníbal. "Coloniality of Power, Eurocentrism, and Social Classification.” Coloniality at Large:Latin America and the Postcolonial Debate, edited by Moraña, Mabel, et al., Duke University Press, 2014, pp. 181-224.

Rivero, Yeidy M. Tuning out Blackness: Race and Nation in the History of Puerto Rican Television. Duke University Press, 2005.

Roy-Féquière, Magali. Women, Creole Identity, and Intellectual Life in Early Twentieth-Century Puerto Rico. Temple University Press, 2004.

Said, Edward W. Culture and Imperialism. Random House, 1993.

---. Orientalism: Edward W. Said. Vintage Books, 1994. Santos-Febres, Mayra. "Contestación a Mayra Montero por su columna racista 'Antes de que llegue el lunes." Facebook. 6 Nov. 2016. esla.facebook.com/permalink. php?story_fbid $=1142254565829780 \& i d=108182309237016$

---. “El color de la seducción.” Sobre piel y papel, por Mayra Santos-Febres, Ediciones Callejón, 2005, pp. 118-122. 